\title{
Mössbauer and SQUID Characterization of Iron in Human Tissue: Case of Globus Pallidus
}

\author{
M. Miglierini ${ }^{a, b, *}$, R. BOČA ${ }^{c}$, M. KOPÁNI ${ }^{d}$, A. $\operatorname{LANČOK}^{e}$ \\ ${ }^{a}$ Institute of Nuclear and Physical Engineering, Faculty of Electrical Engineering and Information Technology, \\ Slovak University of Technology in Bratislava, Ilkovičova 3, 81219 Bratislava, Slovakia \\ ${ }^{b}$ Regional Centre of Advanced Technologies and Materials, Palacky University, \\ 17. listopadu 12, 77146 Olomouc, Czech Republic \\ ${ }^{c}$ Department of Chemistry, FPV, University of SS. Cyril and Methodius, 91701 Trnava, Slovakia \\ ${ }^{d}$ Department of Pathology, Faculty of Medicine, Comenius University, Sasinkova 4, 81108 Bratislava, Slovakia \\ ${ }^{e}$ Institute of Inorganic Chemistry AS CR, v. v. i., 25068 Husinec-Řež 1001, Czech Republic
}

\begin{abstract}
Lyophilized samples of human brain tissue from the region of Globus Pallidus were investigated in powder form by ${ }^{57} \mathrm{Fe}$ Mössbauer spectroscopy and SQUID magnetometry. The obtained hyperfine parameters including isomer shift and quadrupole splitting exhibit a ferritin-like behaviour with high-spin Fe(III) sites. Three types of features were derived from temperature development of magnetic susceptibility: dominating diamagnetism, dominating paramagnetism, and intermediate states. The magnetic characteristics of the samples do not correlate with age of the donors.
\end{abstract}

DOI: $10.12693 /$ APhysPolA.126.240

PACS: 76.80.+y, 31.30.Gs, 75.75.-c, 87.85.jc

\section{Introduction}

Iron is the most important metal with high concentration $(205 \mu \mathrm{g} / \mathrm{g}$ wet mass [1]) in some regions of the brain. It catalyzes reactions forming reactive oxygen species and it is one of the major factors associated with neurodegenerative diseases [2]. Since the iron can exist in organism mainly in two oxidation states (Fe(II) and $\mathrm{Fe}(\mathrm{III})$ ), it is important to identify its oxidation states.

${ }^{57} \mathrm{Fe}$ Mössbauer spectroscopy (MS) is a suitable method that provides information both on structural arrangement and magnetic states of the resonant ${ }^{57} \mathrm{Fe}$ nuclei. MS experiments performed upon parts of human brains showed that most of the iron in the brain is ferritin-like [3, 4]. In order to better identify spectral components of biomedical substances, MS with high velocity resolution was recently introduced [5]. This work characterizes the magnetic behaviour of iron in Globus Pallidus.

\section{Experimental details}

The samples were extracted post mortem from 26 to 85 year old donors (men) according to the Helsinki Declaration. Special attention was paid to avoid manipulations with magnetisable instruments and environment. Fresh, soft tissues were dried in a vacuum (lyophilized). In total, 21 samples were obtained in a form of a powder.

${ }^{57} \mathrm{Fe}$ MS of 17 samples was carried out at room temperature in transmission geometry by a standard constant acceleration spectrometer equipped with a ${ }^{57} \mathrm{Co} / \mathrm{Rh}$

*corresponding author; e-mail: marcel.miglierini@stuba.sk source. Isomer shifts are quoted to bcc-Fe. Magnetic measurements were done by the SQUID magnetometer (Superconducting Quantum Interference Device; Quantum Design, model MPMS-XL7) upon 12 samples.

\section{Results and discussion}

A typical MS spectrum is shown in Fig. 1. The twoline pattern is characteristic for a non-magnetic material that demonstrates at room temperature only the paramagnetism due to the presence of ferritin. All 17 spectra were refined by a single doublet similar as in $[3,6]$.

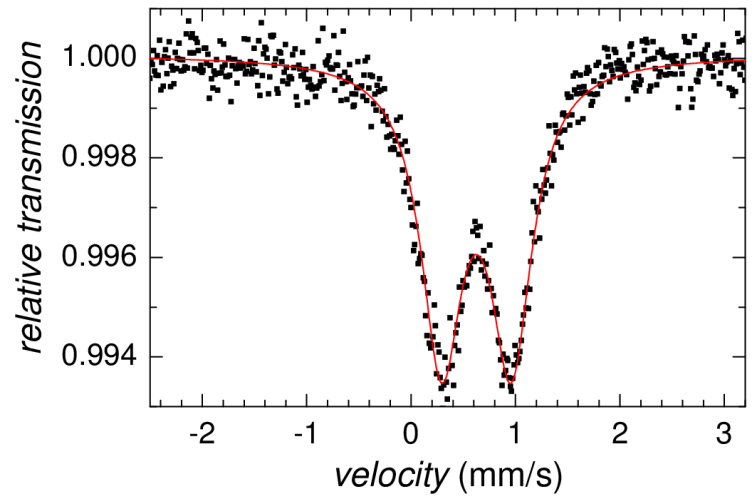

Fig. 1. Typical Mössbauer spectrum of Globus Pallidus.

The obtained plot of quadrupole splitting versus isomer shift in Fig. 2 shows that majority of the spectral parameters can be clearly grouped within one region. Nevertheless, values of 3 samples (open squares) are outside this area. 


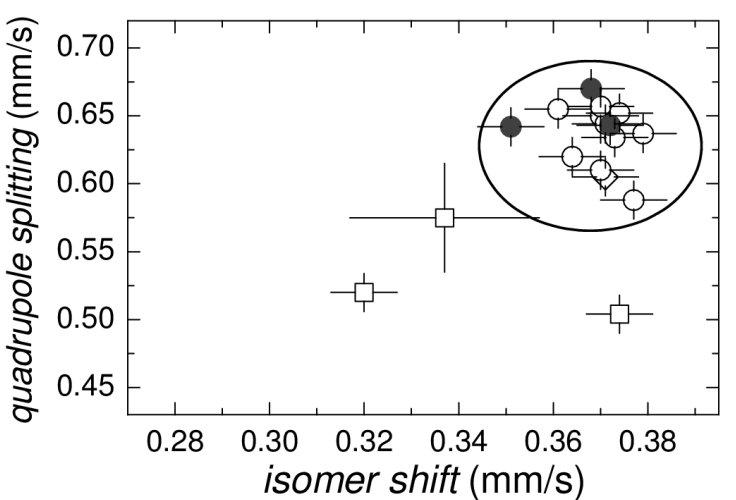

Fig. 2. Quadrupole splitting plotted versus isomer shift of the room temperature MS spectra of brain samples (see text).
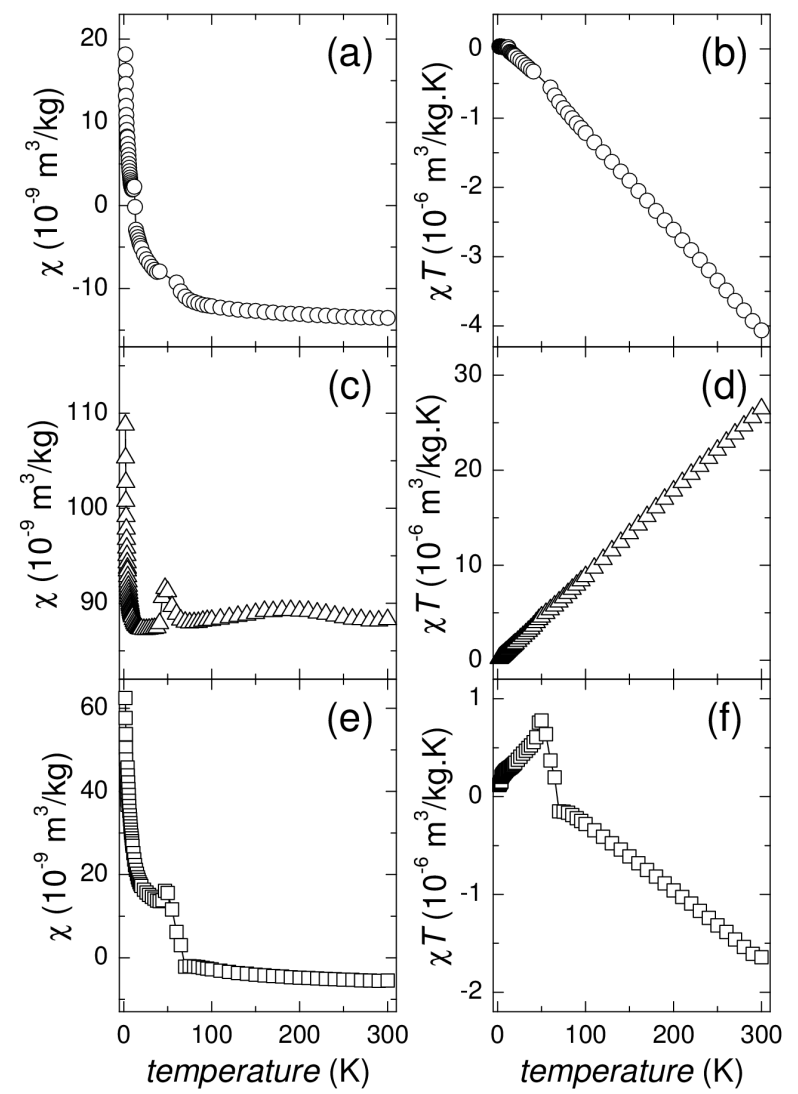

Fig. 3. Magnetic susceptibility $\chi$ (a, c, e) and the product functions $\chi T(\mathrm{~b}, \mathrm{~d}, \mathrm{f})$ plotted against temperature for the Class I $(\mathrm{a}, \mathrm{b})$, Class III $(\mathrm{c}, \mathrm{d})$, and Class II $(\mathrm{e}$, f) samples.

During SQUID experiments the samples were placed into a gelatine-made holder. The obtained temperature dependencies of magnetic susceptibility $\chi($ at $0.1 \mathrm{~T})$ and product functions $\chi T$ can be classified into three classes.

Representative examples that are shown in Fig. 3 correspond to the samples which MS parameters are plotted by solid circles in Fig. 2. All of them fall into the marked area.

In the Class I (Fig. 3a), $\chi$ increases on cooling only slightly. On further cooling, it rises more rapidly. Except of very low temperatures, $\chi T$ shows a negative slope (Fig. 3b). This indicates dominating diamagnetism due to organic tissue with a presence of paramagnetic and/or ferromagnetic centres in 6 samples. Class III (2 samples) is characterized by positive $\chi$ in the whole temperature region (Fig. 3c) and $\chi T$ adopts a positive slope thus proving a dominating para/ferro-magnetism (Fig. 3d). Class II ( 4 samples) is intermediate: $\chi T$ rises with temperature (Fig. 3f) as in the Class III but then reverses sign and behaves as Class I.

\section{Conclusions}

Mössbauer spectrometry indicates that Fe occupies high-spin $\mathrm{Fe}(\mathrm{III})$ states and exhibits a ferritin-like behaviour in the Globus Pallidus. On the other hand, SQUID magnetometry has revealed different magnetic states of the investigated samples. Their origin might be assigned to distribution in size and shape of the Fe nanoparticles [7] that affect also the blocking temperature [8], as we have recently determined for human and horse spleen tissues by MS. Presence of bigger paramagnetic and/or ferromagnetic iron oxide deposits outside ferritin (or its inorganic core) cannot be excluded, too. In the present study, no correlation between the magnetic characteristics of the samples and age of the donors was established.

\section{Acknowledgments}

This work was supported by the research projects VEGA 1/0220/12, P204/10/0035, CZ.1.05/2.1.00/03.0058, and CZ.1.07/2.3.00/20.0155.

\section{References}

[1] C. Langkammer, N. Krebs, W. Goessler, E. Scheurer, F. Ebner, K. Yen, F. Fazekas, S. Ropele, Radiology 257, 455 (2010).

[2] A. Friedman, P. Arosio, D. Finazzi, D. Koziorowski, J. Galazka-Friedman, Parkinsonism and Related Disorders 17, 423 (2011).

[3] J. Galazka-Friedman, A. Friedman, E.R. Bauminger, Hyperfine Interact 189, 31 (2009).

[4] J. Gałazka-Friedman, E.R. Bauminger, K. Szlachta, D. Koziorowski, R. Tomasiuk, A. Jaklewicz, Z.K. Wszołek, D. Dickson, K. Kaplinska, A. Friedman, Acta Phys. Pol. 119, 81 (2011).

[5] M.I. Oshtrakh, V.A. Semionkin, O.B. Milder, E.G. Novikov, J. Mol. Structure 924-926, 20 (2009).

[6] S.M. Dubiel, B. Zablotna-Rypien, J.B. Mackey, J.M. Williams, Eur. Biophys. J. Biophys. Lett 28, 263 (1999).

[7] M. Miglierini, J. Dekan, M. Kopani, A. Lancok, J. Kohout, M. Cieslar, AIP Conf. Proc. 1489, 107 (2012).

[8] M. Miglierini, A. Lancok, Acta. Phys. Pol. A 118, $944(2010)$. 